\title{
Loss of KLF15 accelerates chronic podocyte injury
}

\author{
SEUNG SEOK HAN ${ }^{1,2}$, MI-YEON YU ${ }^{1}$, KYUNG DON YOO $^{3}$, \\ JUNG PYO LEE ${ }^{1,2}$, DONG KI KIM ${ }^{1,2}$, YON SU KIM ${ }^{1,2}$ and SEUNG HEE YANG ${ }^{2}$ \\ ${ }^{1}$ Department of Internal Medicine; ${ }^{2}$ Kidney Research Institute, Seoul National University \\ College of Medicine, Seoul 03080; ${ }^{3}$ Department of Internal Medicine, Dongguk University \\ College of Medicine, Gyeongju, Gyeongsangbuk-do 13620, Republic of Korea
}

Received January 5, 2018; Accepted June 6, 2018

DOI: 10.3892/ijmm.2018.3726

\begin{abstract}
Krüppel-like factor 15 (KLF15), also known as kidney-enriched transcription factor, is known to participate in podocyte differentiation. However, the role of KLF15 in chronic podocyte injury remains incompletely understood, particularly in proteinuric disease models. In the present study, the 5/6 nephrectomy mouse model was used to induce chronic podocyte injury. Human primary podocytes were isolated by flow cytometry and cultured to emulate the injury process in an in vitro system. Biopsied kidney tissue samples were obtained from patients with primary membranous nephropathy or diabetic nephropathy in order to analyze the relationship between glomerular KLF15 expression and subsequent outcomes. When 5/6 nephrectomy was predisposed to progressive kidney damage, fibrosis markers increased, while podocyte KLF15 expression decreased. In addition, increased fibrosis marker expression in human primary podocytes following treatment with transforming growth factor- $\beta$ was aggravated by the knockdown of KLF15. These trends were reversed after cultured podocytes were treated with cyclosporine. When patients were grouped according to KLF15 expression levels in kidney tissue, the low expression groups were demonstrated to have worse renal outcomes, such as non-remission of disease and end-stage renal disease. In conclusion, the present findings revealed that low expression of KLF15 was associated with chronic podocyte injury.
\end{abstract}

\section{Introduction}

Chronic kidney disease (CKD) and its terminal stage, end-stage renal disease, are important health concerns, as both are associated with high morbidity and mortality $(1,2)$. The high global prevalence of CKD is increasing, requiring

Correspondence to: Professor Seung Hee Yang, Kidney Research Institute, Seoul National University College of Medicine, 103 Daehak-ro, Seoul 03080, Republic of Korea

E-mail: ysh5794@gmail.com

Key words: chronic kidney disease, cyclosporine, Krüppel-like factor 15 , podocyte urgent interventions (3). Despite its importance, no established therapeutic approaches exist that can restore the kidney to its healthy state. Therefore, elucidating the pathophysiology of CKD is highly important, as is translating basic research results to clinical practice.

Podocytes form the glomerular filtration barrier, through interactions with endothelial cells and basement membrane components. If podocyte injury occurs or if there is loss to their intercalated structure, glomerular filtration capacity decreases, which results in proteinuria (4). Ongoing podocyte injury is a representative feature of CKD (4), and is attributable to several underlying problems, including primary glomerulonephritis, diabetic nephropathy and other secondary forms (5-7). Notably, podocyte injury is directly associated with patient mortality (8). Therefore, aiming to reduce podocyte injury is a research focus, as current therapeutic options are not fully sufficient. Some of the available options, such as modulators of the renin-angiotensin-aldosterone system, do not target podocyte pathophysiology, while steroids have several side effects following prolonged use (9).

Krüppel-like factor (KLF) is a member of the zinc-finger family of transcription factors, which preferentially binds to GC-rich sequences (for example, CACCC) (10). To date, 17 subclasses have been identified in the human genome, and each regulates diverse and distinct cellular processes. Their expression levels also differ according to the type of tissue. KLF15 is abundant in podocytes (11), hepatocytes, and cardiomyocytes $(12,13)$. Of note, KLF15 regulates the transcription of podocyte-specific genes, such as nephrin and podocin (11). This property may represent a potential novel target that can be manipulated for therapeutic purposes in cases of podocyte injury. A follow-up study supported the hypothesis that KLF15 may serve as a therapeutic target, reporting that dexamethasone, which is used for treating podocyte inflammation, induced KLF15 expression and reduced podocyte injury in a model of focal segmental glomerulosclerosis (14). In a model of hypertensive nephropathy, Sirtuin-3 was demonstrated to be responsible for KLF15 deacetylation, which prevented renal injury (15).

The present study hypothesized that podocyte-specific expression of KLF15 may be altered in CKD. Using both in vitro (primary human podocytes) and in vivo (the 5/6 nephrectomy mouse model) systems, the results revealed that low expression of KLF15 was associated with severe renal injury. The present 
results also demonstrated that cyclosporine, which is known to reduce podocyte injury (16), increased KLF15 expression. Finally, KLF15 expression was examined in biopsied kidney samples from patients, which may serve as a useful biomarker in conditions such as membranous nephropathy and diabetic nephropathy.

\section{Materials and methods}

Mice. All animal experiments were performed under the approval of the Institutional Animal Care and Use Committee of Seoul National University Hospital (Seoul, Korea; approval no. 12-0132), according to the Guide for the Care and Use of Laboratory Animals of the National Research Council. Wild type and C-C chemokine receptor (CCR) type 5-deficient (CCR5-/-) mice were obtained from the Orient Company (Seoul, Korea) and the Jackson Laboratory (Bar Harbor, ME, USA) on a C57BL/6 background. Animals were maintained at $22 \pm 2^{\circ} \mathrm{C}$ with a relative humidity of $40-60 \%$ and a $12 \mathrm{~h}$ light/dark cycle. Animals had ad libitum access to a commercial rodent diet and tap water. A total of 72 adult male mice (7-8 weeks old) were used for the 5/6 nephrectomy model. To produce visible renal ischemia in one-third of the kidney, the lower branch of the right renal artery was suture ligated, and the upper branch of the right kidney was amputated using electrocoagulation. The upper branch of the left renal artery was then ligated, and the lower pole was placed back in the renal fossa by cauterization. Left nephrectomy was performed 1 week later, which indicated the onset of moderate-to-severe kidney injury (day 0). Parameters such as blood pressure, serum blood urea nitrogen, creatinine, and proteinuria were subsequently monitored.

Isolation and culture of primary human podocytes. The study protocol complied with the Declaration of Helsinki and received full approval from the Institutional Review Board at Seoul National University Hospital (approval no. 1607-133-777). Primary human podocytes were obtained in January 2017 from the unaffected pole of a nephrectomy specimen from a patient with renal cell carcinoma (45 years old, male) from the Seoul National University Hospital (Seoul, Korea). Kidney cortices were mechanically dissected. Glomerular cells were sieved using optimized media (17), and isolated by flow cytometry for a podocalyxin-positive (cat. no. FAB1658P; R\&D Systems, Inc., Minneapolis, MN, USA) and CD90-negative (cat. no. 11-0909-41; eBioscience; Thermo Fisher Scientific, Inc., Waltham, MA, USA) population. The purities of sorted cells were $>98 \%$. Subsequent gating analyses were conducted with the FlowJo software (version 10; FlowJo LLC, Ashland, OR, USA). The overall procedure was performed as previously described (18).

For in vitro stimulation, cells were maintained at a concentration of $1 \times 10^{5}$ cells per well in 6-well plates, and starved for $24 \mathrm{~h}$. They were then treated with $1 \mu \mathrm{M}$ retinoic acid (cat. no. R2625; Sigma-Aldrich; Merck KGaA, Darmstadt, Germany) or $2 \mathrm{ng} / \mathrm{ml}$ recombinant transforming growth factor- $\beta$ (TGF- $\beta$ ) for 48 h (R\&D Systems, Inc.). In other experiments, cyclosporine (Calbiochem; Merck KGaA) was used as a renoprotective agent. Small interfering RNA (siRNA) was transfected into cells in order to knockdown the KLF15 expression as suggested in instructor's protocol (cat. no. sc-45567; Santa Cruz Biotechnology, Inc., Dallas, TX, USA). For KLF15 silencing, $1 \mu$ mol human KLF15 siRNA or $1 \mu$ mol scrambled non-targeting siRNA (cat. no. sc-36869) was added.

Briefly, $24 \mathrm{~h}$ prior to transfection, cells were washed, detached with trypsin, diluted to 1:5 with fresh medium without antibiotics $\left(2 \times 10^{5}\right.$ cells $\left./ \mathrm{ml}\right)$, transferred to 6 -well plates $(1 \mathrm{ml} /$ well) and grown to $60-80 \%$ confluency. A mixture of KLF15 siRNA with transfection reagent (cat. no. sc-29528; Santa Cruz Biotechnology) into siRNA transfection medium (cat.no. sc-36868; Santa Cruz Biotechnology) was prepared and incubated for $30 \mathrm{~min}$ at room temperature. Complete medium was subsequently replaced with $1 \mathrm{ml}$ of the above transfection mixture. Approximately $6 \mathrm{~h}$ later, this was replaced with $1 \mathrm{ml}$ normal complete medium. After $24 \mathrm{~h}$, cells were treated with recombinant TGF- $\beta(2 \mathrm{ng} / \mathrm{ml})$ and cyclosporine $(2 \mu \mathrm{mol})$ in fresh complete medium for $48 \mathrm{~h}$. Specific silencing of KLF15 was confirmed using western blot analysis in three independent experiments.

Microscopy imaging analysis. Harvested mouse or biopsied human kidney tissue samples were fixed in $4 \%$ paraformaldehyde at $4^{\circ} \mathrm{C}$ overnight. Paraffin-embedded tissue sections $(4 \mu \mathrm{m})$ were deparaffinized and rehydrated with xylene three times (5 min each) and a descending ethanol series (100, 100, 95, 90, 80 and 70\%; 5 min each), respectively. Endogenous streptavidin activity was blocked in $0.3 \%$ hydrogen peroxide. Antigens were retrieved by heating paraffin-embedded sections to $\sim 95^{\circ} \mathrm{C}$ in $10 \%$ citrate buffer in a microwave oven, five times for 5 min each. Blocking reagent (cat. no. X0909; Agilent; Santa Clara, CA, USA) was incubated with sections at room temperature for $1 \mathrm{~h}$ to block non-specific background staining. The sections were subsequently stained with periodic acid-Schiff and Sirius red (cat. no. ab150681; Abcam, Cambridge, UK), and antibodies against KLF15 (1:100; cat. no. AV32587; Sigma-Aldrich; Merck KGaA), and counterstained with hematoxylin at $4^{\circ} \mathrm{C}$ overnight. Morphometric parameters were captured using a microscope coupled to a computerized morphometry system (Leica Microsystems, Rijswijk, Netherlands). By the use of isotype controls for the anti-KLF15 antibody (1:100; cat. no. A0545; Sigma-Aldrich; Merck KGaA), non-specific staining could be excluded.

Confocal imaging was also performed following staining with DAPI (1:1,000; cat. no. 62248; Thermo Fisher Scientific, Inc.). Blocking reagent (cat. no. X0909; Agilent) was added for $1 \mathrm{~h}$ at room temperature. PBS-diluted antibodies against synaptopodin (1:100; cat. no. 61094; Progen Biotechnik, Heidelberg, Germany), nephrin (1:100; cat. no. PA5-25932; Thermo Fisher Scientific, Inc.), CD31 (1:50; cat. no. ab56299; Abcam), Wilms tumor-1 (1:100; cat. no. ab8990-1; WT-1; Abcam), and KLF15 (1:100; cat. no. AV32587; Sigma-Aldrich; Merck $\mathrm{KGaA}$ ) were subsequently incubated with the sections at $4^{\circ} \mathrm{C}$ overnight. Alexa Fluor 488- (1:400; cat. no. A-10631; Thermo Fisher Scientific, Inc.), Alexa Fluor 555-(1:400; cat. no. A-21433; Thermo Fisher Scientific, Inc.), and Alexa Fluor 647-antibodies (1:400; cat. no. A-21445; Thermo Fisher Scientific, Inc.) were then added at room temperature for 30 min. Confocal microscopy was performed with a Leica TCS SP8 STED CW (Leica Microsystems). 
Western blot analysis. Primary human podocytes were lysed with radioimmunoprecipitation assay lysis buffer (cat. no. 89900; Thermo Fisher Scientific, Inc.) supplemented with complete protease inhibitor (cat. no. 78420; Thermo Fisher Scientific, Inc.). The kidney homogenate was centrifugated at $12,000 \mathrm{x} \mathrm{g}$ and $4^{\circ} \mathrm{C}$ for $30 \mathrm{~min}$. Protein concentration of the supernatant was determined with the Bradford method. Equal amounts $(80 \mu \mathrm{g})$ of extracted protein were separated by $10 \%$ SDS-PAGE and transferred onto Immobilon-FL $0.4 \mu \mathrm{M}$ polyvinylidene difluoride membranes (Millipore, Bedford, MA, USA). Blocking was conducted at room temperature for $1 \mathrm{~h}$ with blocking buffer $(5 \%$ skin milk and $2 \%$ bovine serum albumin (cat. no. 10735086001; Sigma-Aldrich; Merck KGaA) in PBS with $0.2 \%$ Tween 20 ). The membranes were then incubated with antibodies against fibronectin (1:300; cat. no. sc-6952; Santa Cruz Biotechnology, Inc.), zonula occludens-1 (ZO-1; 1:300; cat. no. 339100; Invitrogen; Thermo Fisher Scientific, Inc.), WT-1 (1:400; cat. no. sc-192; Santa Cruz Biotechnology, Inc.), $\beta$-actin (1:10,000; cat. no. a5316; Sigma-Aldrich; Merck $\mathrm{KGaA}$ ), and KLF15 (1:200; cat. no. ab167192; Abcam) at $4^{\circ} \mathrm{C}$ overnight. Horseradish peroxidase-conjugated secondary anti-rabbit (1:4,000; cat. no. 7074S; Cell Signaling Technology, Inc. Danvers, MA, USA), anti-goat (1:4,000; cat. no. sc-2005; Santa Cruz Biotechnology, Inc.), and anti-mouse (1:4,000; cat. no. 7076S; Cell Signaling Technology, Inc.) immunoglobulin $\mathrm{G}$ antibodies were added for $1 \mathrm{~h}$ at room temperature. The blots were developed and enhanced using a Super Signal West Pico Chemiluminescence kit. Band intensity was assessed using ImageJ software (version 1.5; National Institutes of Health, Bethesda, MD, USA).

Reverse transcription-quantitative polymerase chain reaction $(R T-q P C R)$. Total RNA was extracted from primary human cells and mouse renal tissue with an RNeasy Micro kit (Qiagen, GmbH, Hilden, Germany). Subsequently, RNA was converted to cDNA using a kit according to the manufacturer's instructions (cat. no. A3500; Promega Corporation, Madison, WI, USA). The TaqMan gene expression assay was used for human fibronectin and GAPDH (Applied Biosystems; Thermo Fisher Scientific, Inc.). The SYBR Green expression assay was used for mouse fibronectin, interleukin (IL)-6, monocyte chemotactic protein (MCP)-1, IL-10, CCR5, KLF15, and GAPDH (Applied Biosystems; Thermo Fisher Scientific, Inc.). Primer sequences were as follows: Mouse fibronectin, forward 5'-ATGCAACGATCAGGACAC AA-3' and reverse 5'-TGTGCCTCTCACACTTCCAC-3'; mouse IL-6, forward 5'-CCTCTGGTCTTCTGGAGTACC-3' and reverse 5'-CGACCTCAGTGTCTTCCTCA-3'; mouse MCP-1, forward 5'-GCTCAGCCAGATGCAGTTAA-3' and reverse 5'-TCAAAAACAGTGGTTCGAGTTCT-3'; mouse IL-10, forward 5'-ATAACTGCACCCACTTCCCA-3' and reverse 5'-TGGACCATCTTCACTACGGG-3'; mouse CCR5, forward 5'-TTGTCTACTTTCTCTTCTGG-3' and reverse 5'-ATCGGGTATAGACTGAGC-3'; mouse KLF15, forward 5'-CACCAAGAGCAGCCACCTCA-3' and reverse 5'-CGG GACACTGGTACGGCTTC-3'; mouse GAPDH, forward 5'-CACCAAGAGCAGCCACCTCA-3' and reverse 5'-CGG GACACTGGTACGGCTTC-3'. Thermocycling conditions were as follows: $95^{\circ} \mathrm{C}$ for $10 \mathrm{~min}$ followed by 40 cycles of dissociation $\left(95^{\circ} \mathrm{C}\right.$ for $\left.10 \mathrm{sec}\right)$, annealing $\left(55^{\circ} \mathrm{C}\right.$ for $\left.30 \mathrm{sec}\right)$, and elongation $\left(72^{\circ} \mathrm{C}\right.$ for $\left.30 \mathrm{sec}\right)$. Data were quantified using the comparative $\mathrm{Cq}\left(2^{-\Delta \Delta \mathrm{Cq}}\right)$ method (19).

Patient sample analysis. Biopsy samples from human kidney tissue were obtained from patients with primary membranous nephropathy $(n=26 ; 27-76$ years old; male, $69.2 \%)$ or diabetic nephropathy $(n=21$; age, 24-67 years old; male, 61.9\%) from the Seoul National University Hospital (Seoul, Korea) between April 2013 and April 2015. Glomerular KLF15 expression was quantified microscopically, and its association with clinical outcomes was analyzed. The clinical outcomes that were analyzed for membranous nephropathy and diabetic nephropathy were spontaneous remission and end-stage renal disease, respectively.

Statistical analysis. All analyses and calculations were performed using SPSS (version 23.0; IBM Corp., Armonk, NY, USA) and GraphPad Prism (version 5.0; GraphPad Software, Inc., La Jolla, CA, USA). The results are expressed as mean \pm standard error of mean. Differences between groups in the western blot, RT-qPCR, and immunohistochemistry analyses were evaluated using one-way analysis of variance, followed by the least significant difference post hoc test. Survival curves were drawn using the Kaplan-Meier method. To compare survival curves between groups, the log-rank test was applied. $\mathrm{P}<0.05$ was considered to indicate a statistically significant difference.

\section{Results}

Podocyte KLF15 expression in the chronic kidney injury model. The 5/6 nephrectomy model was used to induce chronic podocyte injury. Additionally, CCR5-/- mice were used to induce podocyte injury with increased severity through alteration of macrophage composition (20). Twenty weeks post-injury, kidney markers such as blood urea nitrogen, creatinine, and the urine protein-to-creatinine ratio were increased in nephrectomized mice compared with sham-operated mice (Fig. 1A). Other surrogate markers, such as systolic blood pressure and body weight exhibited significant differences between nephrectomized and sham-operated mice (Fig. 1A). When these markers were compared between nephrectomized wild type and CCR5-/- mice, aggravated injury was observed in the CCR5-/- mice. In specific, the measurements were: Body weight $(\mathrm{g}), 26.1 \pm 0.44$ vs. $24.5 \pm 0.33$; blood urea nitrogen $(\mathrm{mg} / \mathrm{dl}), 51.8 \pm 3.10$ vs. $70.0 \pm 4.21$; creatinine $(\mathrm{mg} / \mathrm{dl}), 0.58 \pm 0.03$ vs. $0.77 \pm 0.04$; and urine protein-to-creatinine ratio $(\mathrm{mg} / \mathrm{mg})$, $13.9 \pm 0.97$ vs. $17.6 \pm 1.00$ in wild type and CCR5-/- mice, respectively (Fig. 1A). Fibronectin, a fibrosis marker, was expressed in higher levels in nephrectomized mice, but podocyte KLF15 expression was decreased and correlated with the degree of injury (Fig. 1B-1D). Production of inflammatory cytokines, IL-6 and MCP-1, was significantly increased (Fig. 1D), while IL-10 production was decreased depending on the degree of podocyte injury (wide type vs. CCR5-/- mice; Fig. 1D). These results suggest that chronic podocyte injury is associated with low KLF15 expression.

KLF15 expression is altered by a renoprotective agent in primary human podocytes. Next, we aimed to confirm that 
A

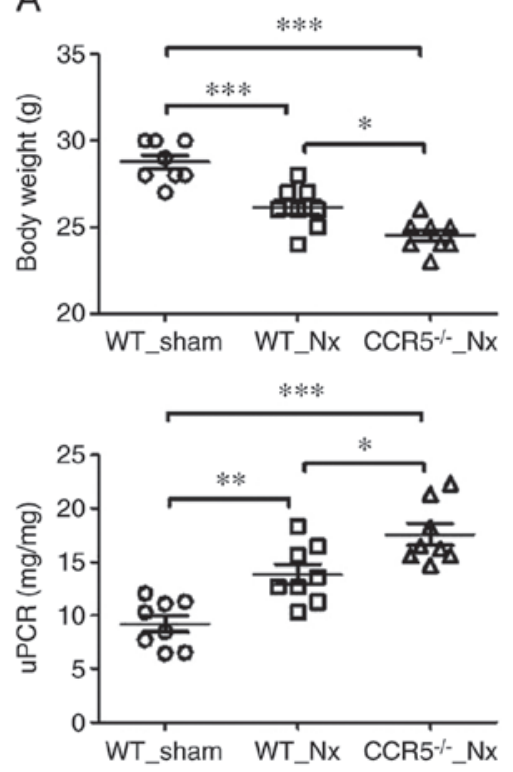

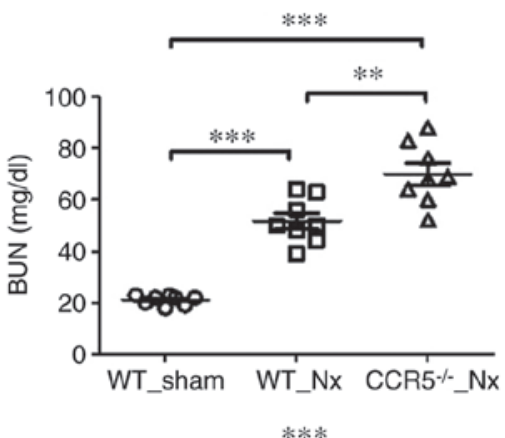

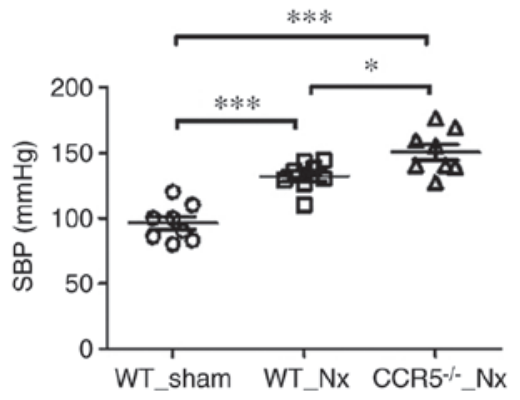

$5 / 6$ Nephrectomy

B
WT

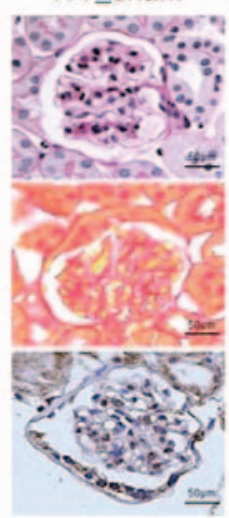

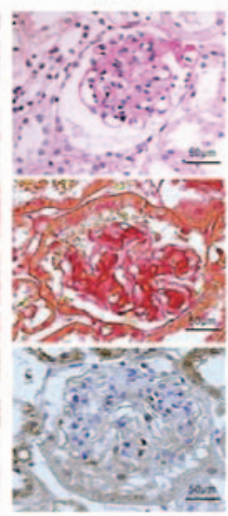

CCR5 $\%$

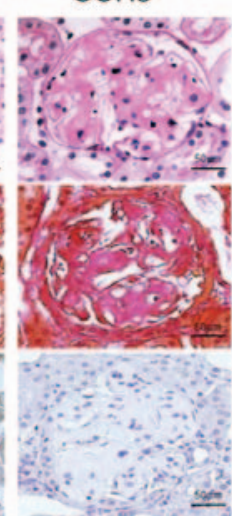

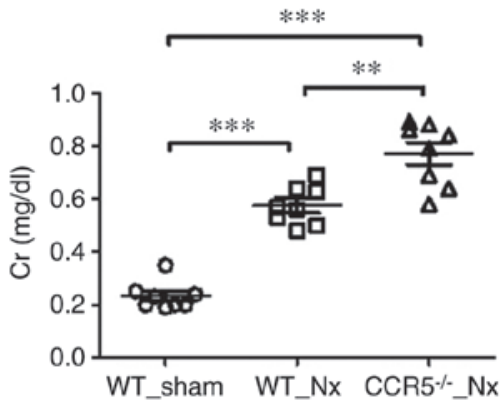

PAS

Sirius red

KLF15

Figure 1. KLF15 expression in an in vivo model of chronic podocyte injury. (A) Renal injury and systemic markers at 20 weeks following $5 / 6$ nephrectomy. Each parameter was evaluated in eight mice per group. (B) Periodic acid-Schiff staining and immunohistochemistry for fibrosis (Sirius red) and KLF15 (magnification, $\mathrm{x} 400$ ). The experiments were performed with eight mice per group. ${ }^{*} \mathrm{P}<0.05,{ }^{* *} \mathrm{P}<0.01$ and ${ }^{* * * *} \mathrm{P}<0.001$, with comparisons indicated by brackets. KLF15, Krüppel-like factor 15; IL, interleukin; MCP-1, monocyte chemotactic protein-1; CCR5, C-C chemokine receptor type 5; WT, wild-type; Nx, 5/6 nephrectomy; BUN, blood urea nitrogen; Cr, creatinine; uPCR, urine protein to creatinine ratio; SBP, systolic blood pressure; PAS, periodic acid-Schiff.

low KLF15 expression is associated with chronic podocyte injury using an in vitro model of primary human cells. Primary human podocytes were isolated and demonstrated to express synaptopodin and nephrin as podocyte markers, while they were negative for the endothelial cell marker, CD31 (data not shown). TGF- $\beta$ is a potent inducer of fibrosis (21), and therefore it was used to induce podocyte fibrosis in vitro. Results from western blot analysis confirmed that fibronectin levels as a fibrosis marker increased when cells were treated with TGF- $\beta$ for $48 \mathrm{~h}$ (Fig. 2).

Retinoic acid and glucocorticosteroids are known to induce the expression of KLF15 in podocytes $(11,14)$. These observations suggest other renoprotective agents may regulate KLF15 expression as part of their protective function. Cyclosporine, a calcineurin inhibitor, was evaluated in the present study, because it is routinely administered to patients with podocytopathy in clinical practice (22). Compared with vehicle-treated cells, cells treated with cyclosporine displayed increased levels of KLF15 and decreased levels of fibronectin that were comparable with the positive control group treated with retinoic acid (Fig. 2A-C). siRNA was then used to knockdown expression of KLF15 in the cells. Following siRNA transfection, the protein expression levels of KLF15 were successfully decreased (Fig. 2D). The siRNA-treated cells exhibited lower ZO-1 and higher fibronectin expressions compared with scramble-treated cells, following TGF- $\beta$ treatment (Fig. $2 \mathrm{E}$ and F). The renoprotective effect of cyclosporine was not evident in the KLF15-knockdown cells (Fig. 2E and F). Cyclosporine could maintain podocytes in a healthier state throughout enrichment of KLF15, in addition to the previous knowledge regarding cytoskeleton stabilization (16).

KLF15 levels and their relationship with clinical outcomes. Previous studies have demonstrated that the decreased KLF15 expression in biopsied kidney tissue from patients with minimal change disease and focal segmental 
C

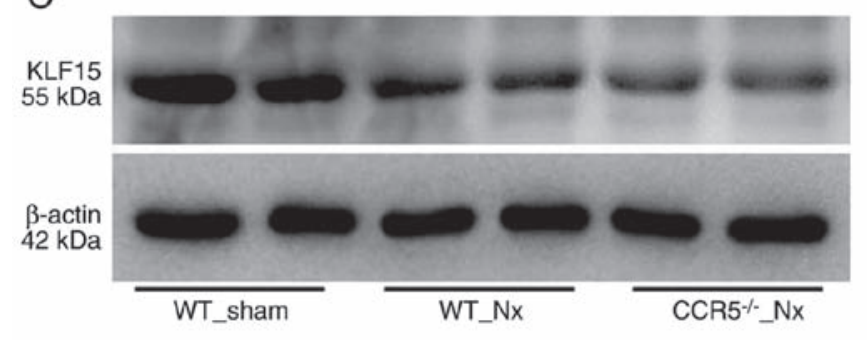

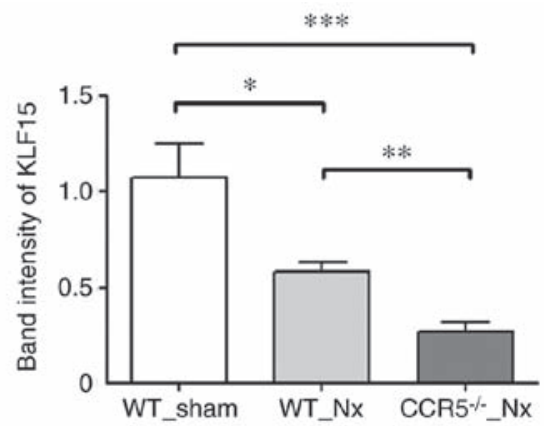

D
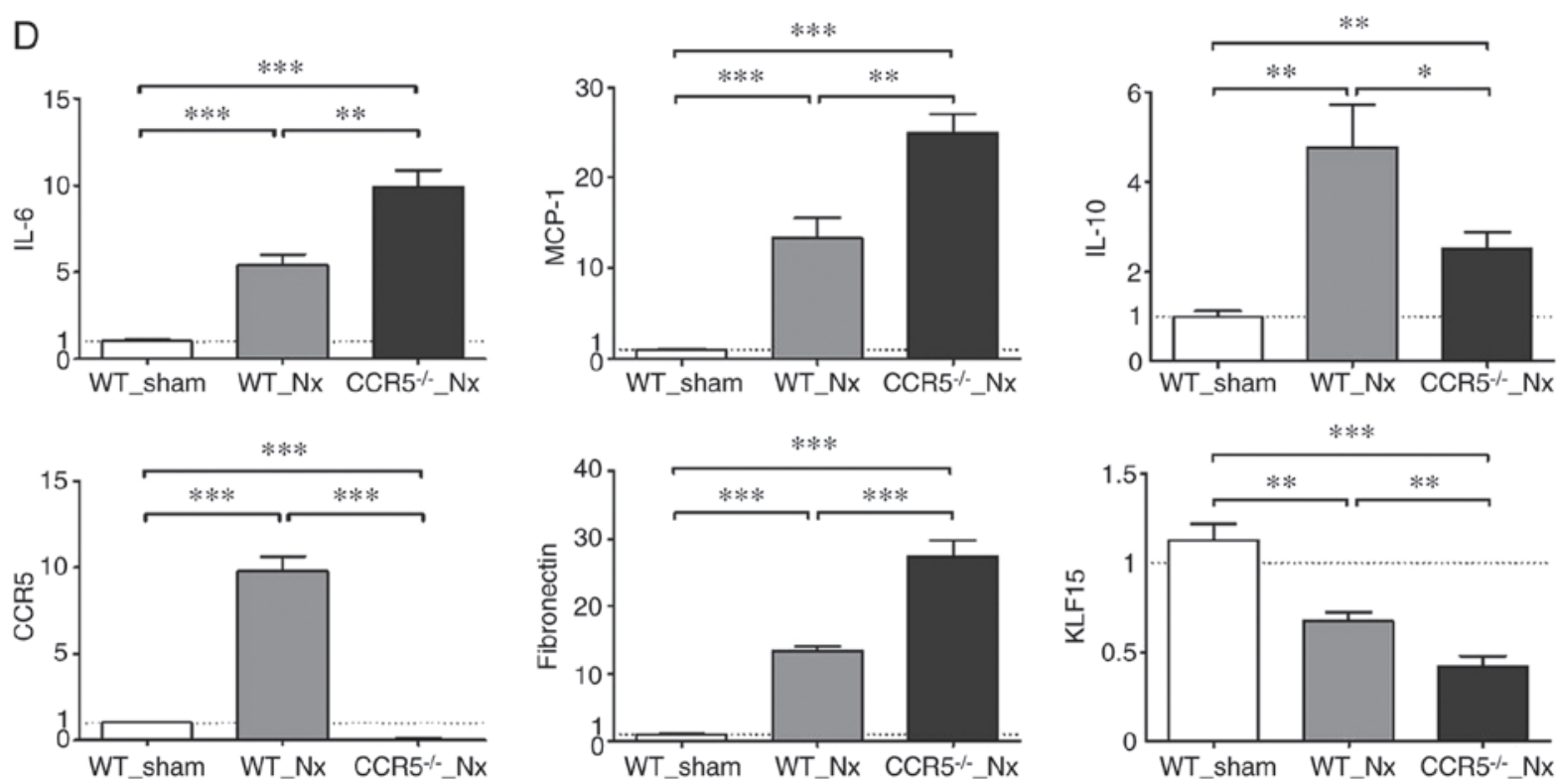

Figure 1. Continued. KLF15 expression in an in vivo model of chronic podocyte injury. (C) Representative western blots and quantitative band intensity analysis for KLF15 expression levels. (D) Quantitative polymerase chain reaction analysis for the mRNA expression levels of fibronectin, IL-6, MCP-1, IL-10, CCR5 and KLF15 from total kidney extracts. The experiments were performed with eight mice per group. ${ }^{*} \mathrm{P}<0.05,{ }^{* *} \mathrm{P}<0.01$ and ${ }^{* * * *} \mathrm{P}<0.001$, with comparisons indicated by brackets. KLF15, Krüppel-like factor 15; IL, interleukin; MCP-1, monocyte chemotactic protein-1; CCR5, C-C chemokine receptor type 5; WT, wild-type; Nx, $5 / 6$ nephrectomy; BUN, blood urea nitrogen; Cr, creatinine; uPCR, urine protein to creatinine ratio; SBP, systolic blood pressure; PAS, periodic acid-Schiff

glomerulonephritis was associated with low response to glucocorticosteroids (14). In the present study, immunohistochemistry results demonstrated that decreased KLF15 expression was predominant in segmental sclerotic glomeruli (Fig. 3A). Next, we aimed to explore the relationship between KLF15 expression and renal outcomes in other podocytopathic diseases, such as membranous nephropathy and diabetic nephropathy. Regarding membranous nephropathy, cases with spontaneous remission (i.e. no use of immunosuppressive drugs) exhibited higher KLF15 expression compared with cases without spontaneous remission (Fig. 3B and C). However, KLF15 expression did not differ between the remission and non-remission cases following administration of immunosuppressive drugs (Fig. 3C). Regarding diabetic nephropathy, the patients were divided into three groups according to KLF15 expression levels (i.e. tertiles), and their renal function was followed for 30 months. The third tertile group (highest expression) did not exhibit doubling of serum creatinine $(\mathrm{P}=0.020)$ or end-stage renal disease $(\mathrm{P}=0.039$; Fig. 3D and E). These results indicate that KLF15 expression is associated with renal outcomes commonly observed in several podocytopathic diseases.

\section{Discussion}

Current therapeutic regimens cannot reverse chronic podocyte injury, and the prevalence and socioeconomic impact of CKD are increasing significantly $(23,24)$. This may be partially because the underlying pathophysiology of CKD is not fully understood. The present study focused on podocyte KLF15 expression in models of CKD. Injured podocytes in 5/6 nephrectomized mice exhibited decreased levels of KLF15. This effect was aggravated in a severe form of podocyte injury (CCR5-/- mice), while it was reverted to high KLF15 expression and low fibrotic phenotype in response to cyclosporine treatment. The present findings suggest that KLF15 may serve as a biomarker related to renal outcomes in patients with podocytopathy.

The KLF family of transcription factors has C-terminal zinc fingers. Since the initial discovery (25), 16 additional proteins have been added to the family. Among them, KLF15 is highly expressed in podocytes and binds the promoter of podocyte-specific genes, such as nephrin and podocin $(11,26)$. Both nephrin and podocin are key components of the filtration slits of podocytes. Mutations in these genes are a representative disorder of congenital nephrotic 

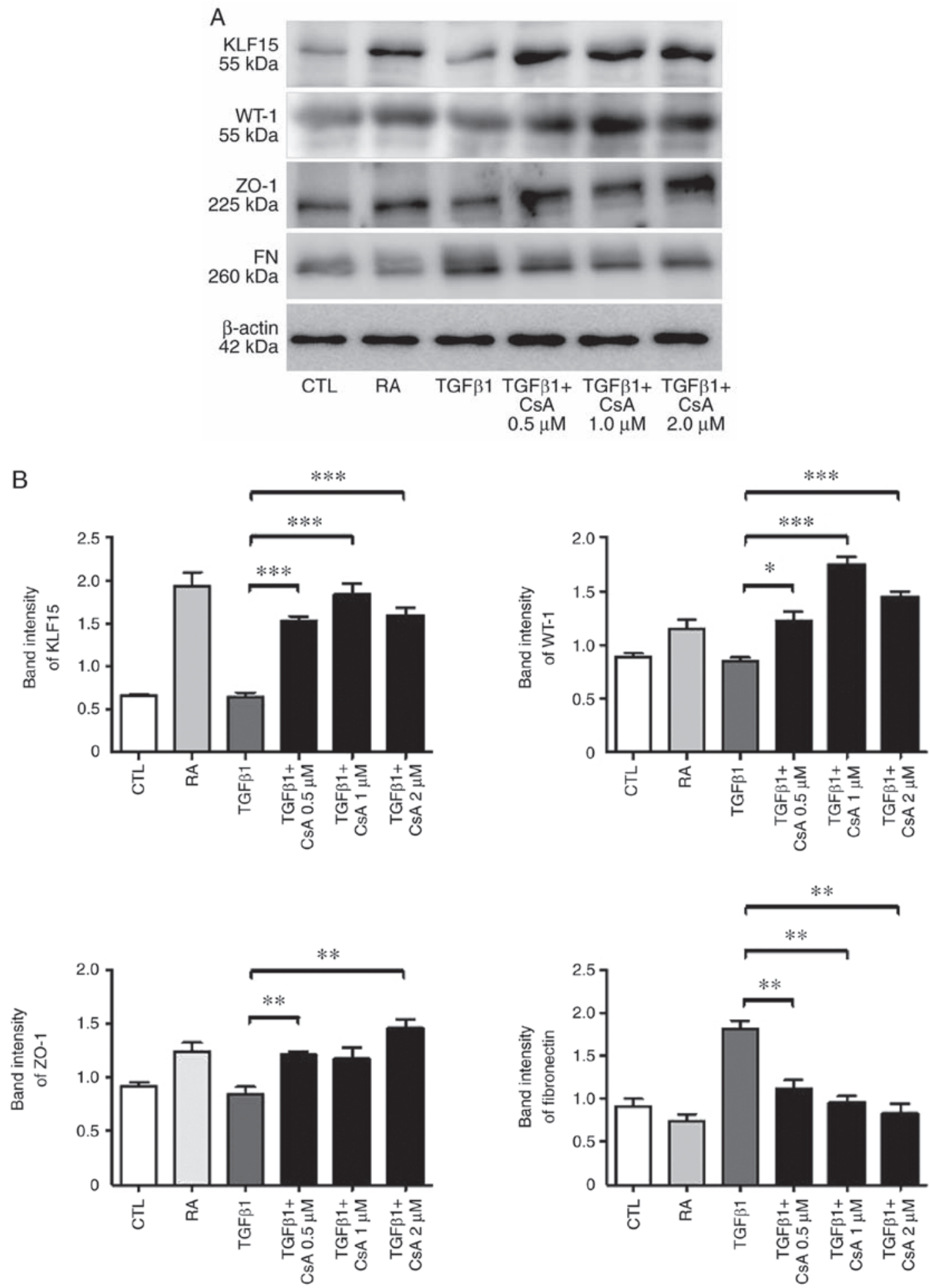

Figure 2. Renoprotective agent-induced changes in podocyte KLF15 expression. (A) Representative western blots for KLF15, WT-1, ZO-1, and fibrosis markers (B) Quantitative analysis of band intensities from the western blot analysis. The experiments were independently repeated three times. ${ }^{*} \mathrm{P}<0.05$, ${ }^{* *} \mathrm{P}<0.01$ and ${ }^{* * * *} \mathrm{P}<0.001$, with comparisons indicated by brackets. KLF15, Krüppel-like factor 15; WT-1, Wilms tumor-1; ZO-1, zonula occludens-1; si, small interfering; TGF- $\beta$, transforming growth factor $\beta ;$; FN, fibronectin; CTL, control; RA, retinoic acid; CsA, cyclosporine.

syndrome $(27,28)$. If these proteins are not successfully produced, the glomerular filtration barrier cannot be maintained and the resultant sign is proteinuria. Accordingly, low or deficient KLF15 expression of podocytes might reduce nephrin and podocin production and subsequently lead to broken glomerular filtration barrier, detachment of podocytes from the glomerular basement membrane, and progressive glomerular injuries. These processes are collectively known as podocytopathies (29). Therefore, KLF15 may have podocyte-specific functions, such as in differentiation and treatment response $(11,14)$. Previous studies have used several murine models, such as lipopolysaccharide- or adriamycin-induced proteinuric models. In the present study, the 5/6 nephrectomy mouse was used as model of chronic 

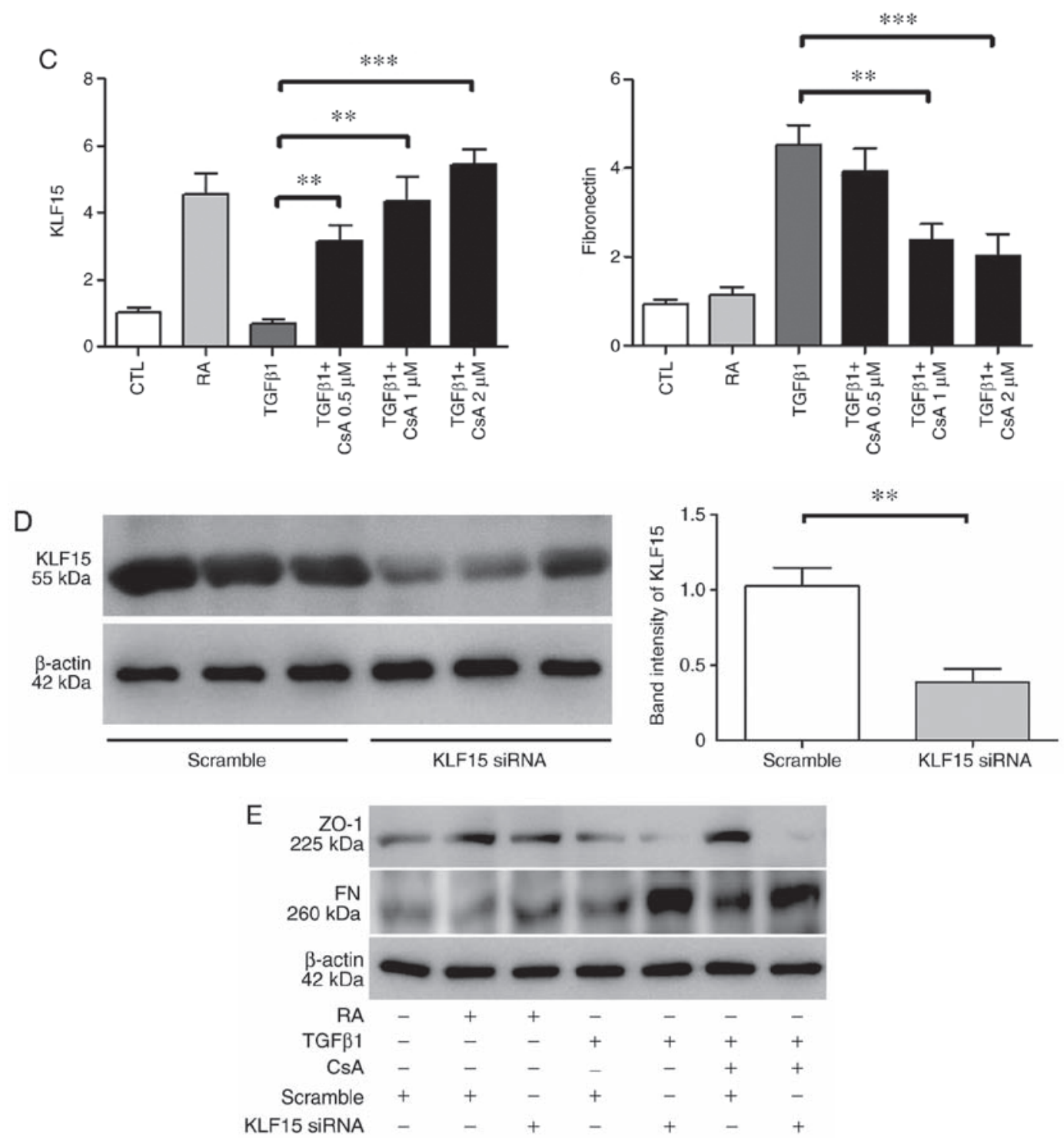

$\mathrm{F}$
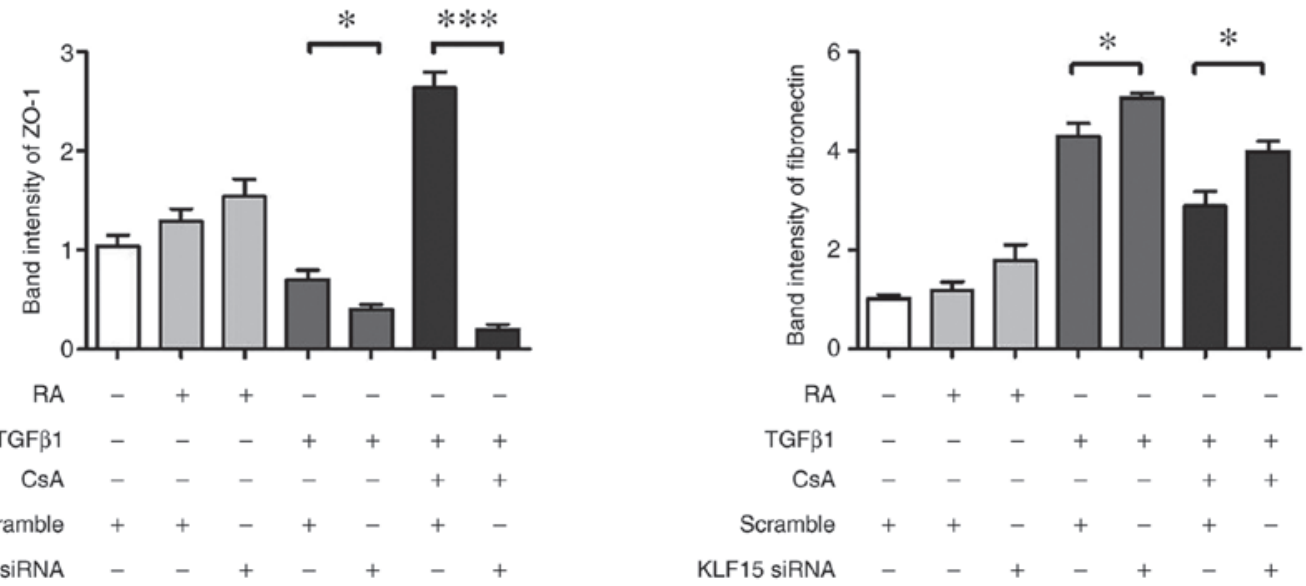

Figure 2. Continued. Renoprotective agent-induced changes in podocyte KLF15 expression. (C) Quantitative polymerase chain reaction analysis for the mRNA expression levels of KLF15 and fibronectin. (D) Representative western blots and quantitative band intensity analysis for KLF15 protein expression levels in scramble- or KLF15 siRNA-treated podocytes. (E and F) Representative western blots and quantitative band intensity analysis for the protein expression levels of ZO-1 and fibronectin in cells treated with retinoic acid, TGF- $\beta$, cyclosporine $(2 \mu \mathrm{M})$, scramble and/or KLF15 siRNA. The experiments were independently repeated three times. ${ }^{*} \mathrm{P}<0.05,{ }^{* *} \mathrm{P}<0.01$ and ${ }^{* * * *} \mathrm{P}<0.001$, with comparisons indicated by brackets. KLF15, Krüppel-like factor 15; WT-1, Wilms tumor-1; ZO-1, zonula occludens-1; si, small interfering; TGF- $\beta$, transforming growth factor- $\beta$; FN, fibronectin; CTL, control; RA, retinoic acid; CsA, cyclosporine.

podocyte injury. Furthermore, primary human podocytes were used in vitro to confirm the results. In specific, silencing of KLF5 by siRNA resulted in an increase of podocyte injury markers. This might be because the structural contexts 
A
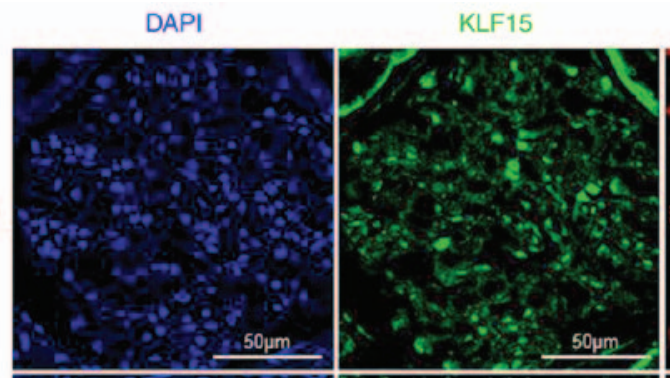

Podocalyain
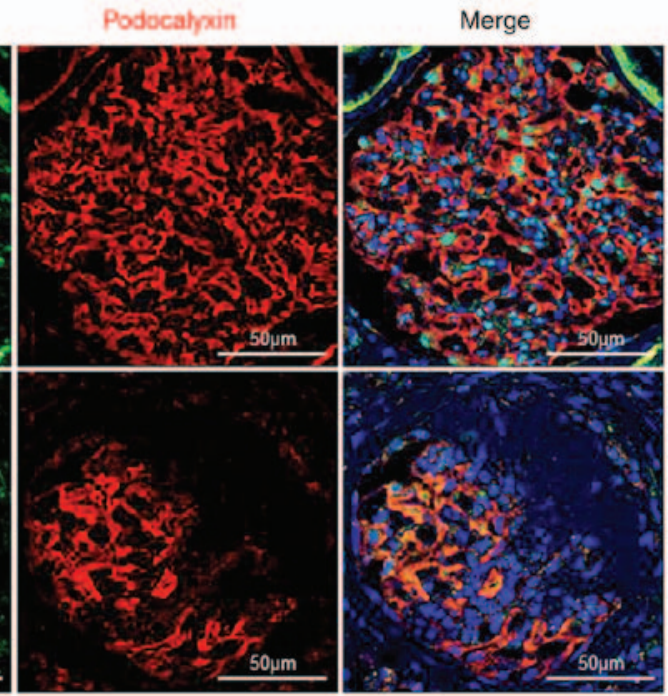

B
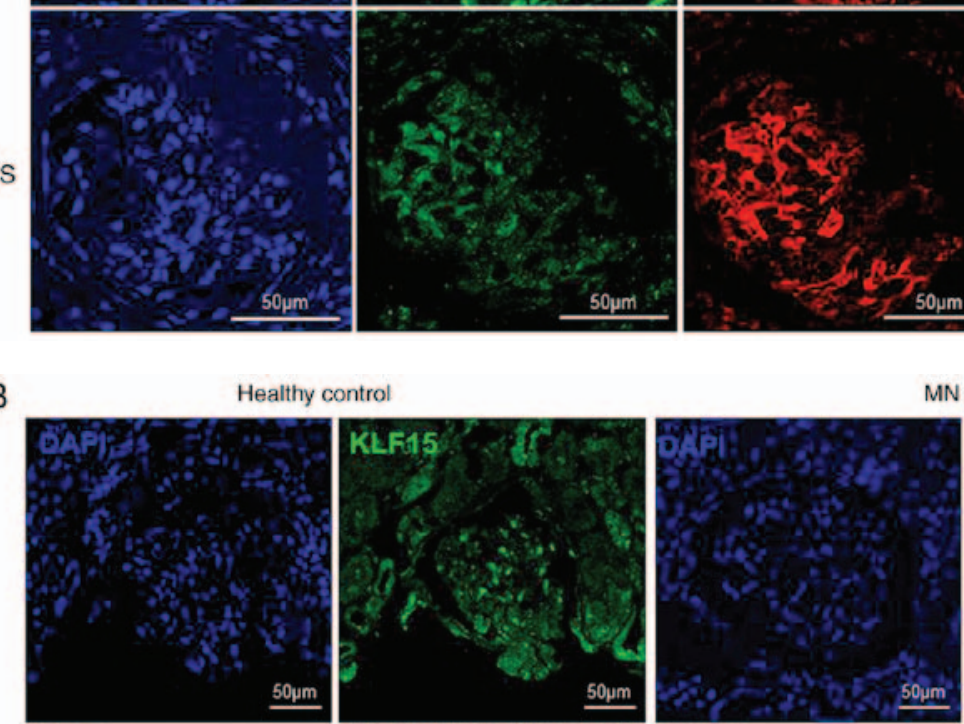

MN patient
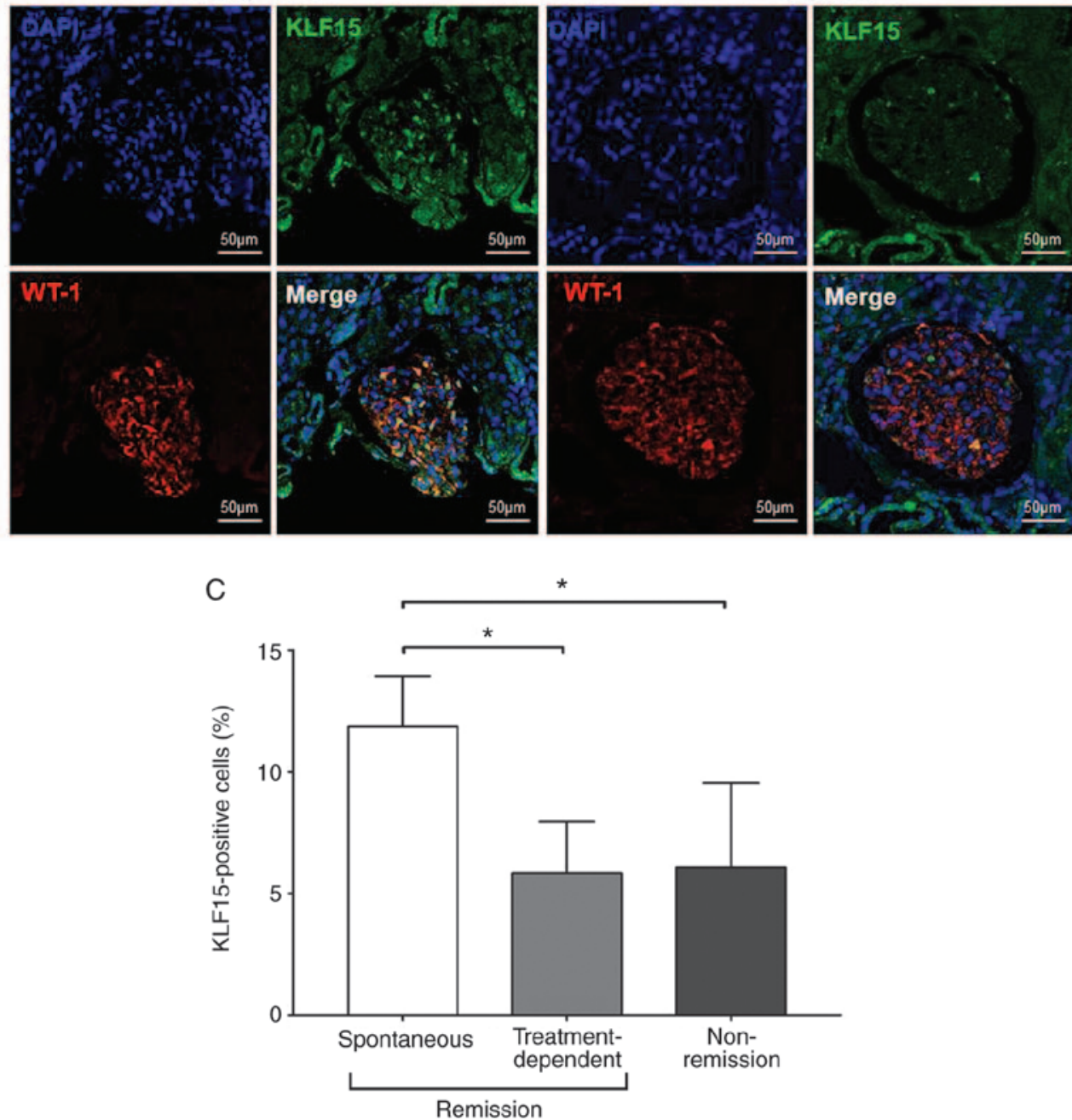

Figure 3. KLF15 expression in biopsied human kidney tissue samples. (A) Representative confocal images of KLF15 and podocalyxin staining in sections of a glomerulus from a healthy control and a patient with focal segmental glomerulosclerosis (magnification, x600). (B) Immunofluorescent images of KLF15 and WT-1 staining of samples from a healthy control and a patient with primary membranous nephropathy (magnification, x400). (C) Association of KLF15 expression levels with spontaneous remission of membranous nephropathy. ${ }^{*} \mathrm{P}<0.05,{ }^{* *} \mathrm{P}<0.01$ and ${ }^{* * *} \mathrm{P}<0.001$, with comparisons indicated by brackets. KLF15, Krüppel-like factor 15; WT-1, Wilms tumor-1; FSGS, focal segmental glomerulosclerosis; DN, diabetic nephropathy; MN, membranous nephropathy; ESRD end-stage renal disease.

including nephrin and podocin were not appropriately maintained and cells were transformed to fibrotic phenotype by depletion or low production of KLF15. These results support the hypothesis that KLF15 is associated with podocyte func- 

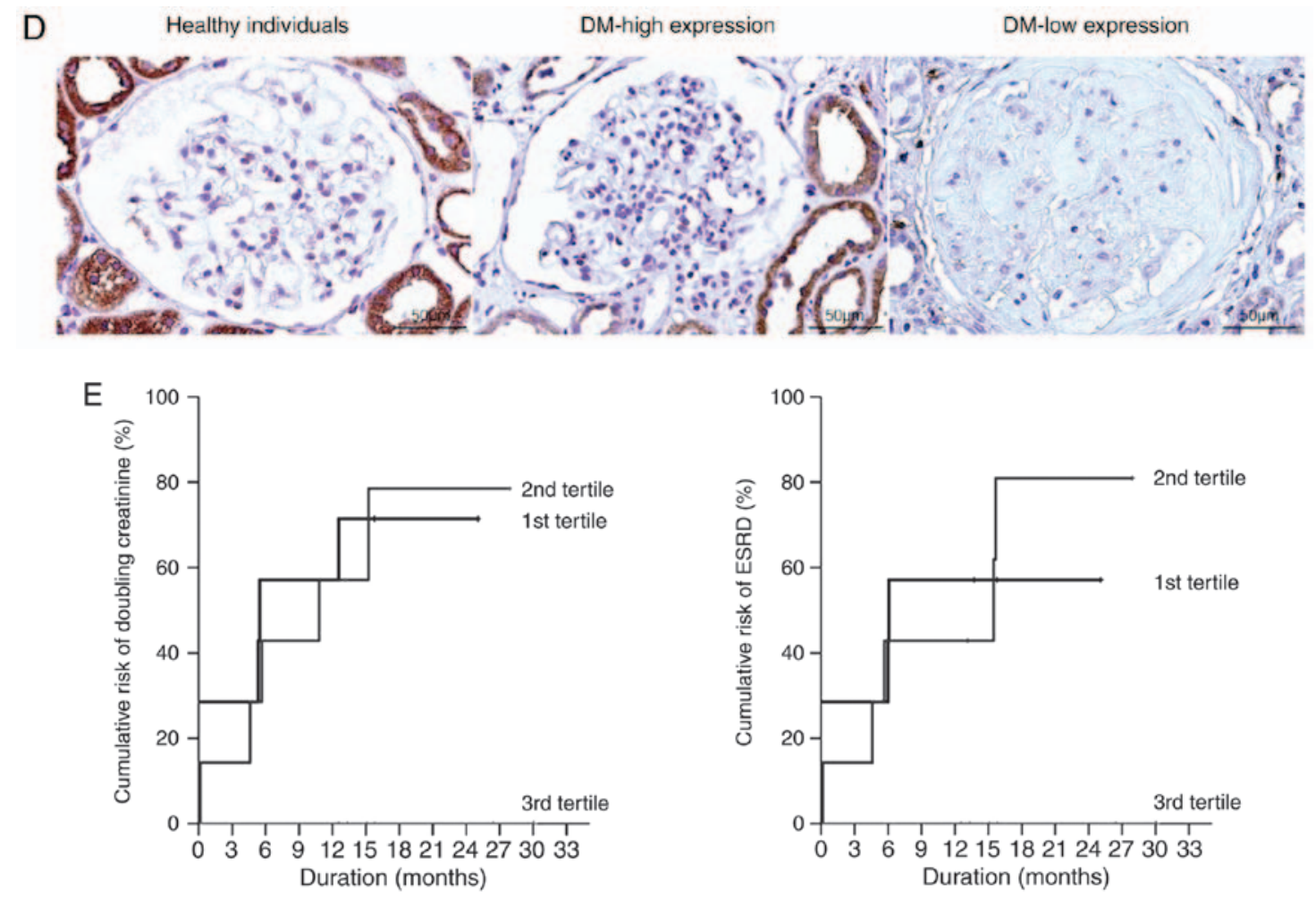

Figure 3. Continued. KLF15 expression in biopsied human kidney tissue samples. (D) Representative images from immunohistochemical staining for KLF15 of samples from healthy control and patients with diabetic nephropathy (magnification, x400). (E) Association of KLF15 expression levels with doubling of serum creatinine and the risk of end-stage renal disease following kidney biopsy. ${ }^{*} \mathrm{P}<0.05,{ }^{* *} \mathrm{P}<0.01$ and ${ }^{* * *} \mathrm{P}<0.001$, with comparisons indicated by brackets. KLF15, Krüppel-like factor 15; WT-1, Wilms tumor-1; FSGS, focal segmental glomerulosclerosis; DN, diabetic nephropathy; MN, membranous nephropathy; ESRD end-stage renal disease.

tion in several disease models, despite differences in the mechanism of podocyte injury.

Current therapeutic regimens for chronic kidney injury, especially those that include management of comorbidities, cannot halt the progression of proteinuria. This is in part because the underlying mechanisms of podocyte injury are incompletely understood. Nonetheless, agents such as glucocorticosteroids and calcineurin inhibitors have been recommended for clinical use. A previous study demonstrated the effect of glucocorticosteroids on the expression of KLF15 in patients with focal segmental glomerulosclerosis, and corresponding murine models (14). The present study also demonstrated the potential role of cyclosporine (a representative calcineurin inhibitor) in the podocyte injury model, by increasing KLF15 expression. The beneficial effect of cyclosporine was diminished in KLF15-knockdown cells, which indicates that KLF may be one of mediators between cyclosporine and podocyte homeostasis. This is a novel function of cyclosporine, although the direct mechanism could not be fully explored in the present study. Recently, resveratrol was also demonstrated to alter KLF15 expression, and to improve outcomes in an ischemic heart model (30). It can be speculated that several other agents that induce KLF15 expression will be identified, and may be used therapeutically for maintaining podocyte homeostasis.

The mechanisms of podocyte injury may differ between different renal diseases. However, based on the observation that KLF15 is highly expressed in podocytes, the loss of KLF15 may be a common late event following initial injury.
The present study aimed to verify this hypothesis in patients with membranous nephropathy and diabetic nephropathy (the most common podocyte diseases in proteinuric adult patients). KLF15 expression was related to clinical outcomes, such as disease remission and progression. These results suggest that KLF15 may serve as a useful biomarker in tissue biopsies.

The present results further validate the association between low KLF15 expression and chronic podocyte injury, which is a typical feature of CKD. Furthermore, altered KLF15 expression may be predictive of outcomes of several podocytopathic diseases. Therapeutic agents that target podocytes are needed in the clinic, ideally ones applicable for several different diseases. In this aspect, future therapies that target KLF15 may be valuable in the clinic.

\section{Acknowledgements}

Not applicable.

\section{Funding}

This work was supported by a grant from the Korea Healthcare Technology R\&D Project, Ministry of Health and Welfare, Republic of Korea (grant no. A111336). Biospecimens were provided by the Seoul National University Hospital Human Biobank, a member of the National Biobank of Korea, which is supported by the Ministry of Health and Welfare, Republic of Korea. 


\section{Availability of data and materials}

The analyzed datasets generated during the study are available from the corresponding author on reasonable request.

\section{Authors' contributions}

SSH designed the study, collected, analyzed and interpreted the data, performed the experiments and drafted the manuscript. MYY and KDY performed the experiments. JPL and DKK collected the data. YSK designed the study. SHY designed the study, performed the experiments, interpreted the data and reviewed the final manuscript. All authors read and approved the manuscript.

\section{Ethics approval and consent to participate}

All experiments involving animals were performed under the approval of the Institutional Animal Care and Use Committee of Seoul National University Hospital (Seoul, Korea; approval no. 12-0132). The experiments involving human tissues complied with the Declaration of Helsinki and received full approval from the Institutional Review Board at Seoul National University Hospital (approval no. 1607-133-777).

\section{Patient consent for publication}

Not applicable.

\section{Competing interests}

The authors declare that they have no competing interests.

\section{References}

1. Schiffrin EL, Lipman ML and Mann JF: Chronic kidney disease: Effects on the cardiovascular system. Circulation 116: 85-97, 2007.

2. Tonelli M, Wiebe N, Culleton B, House A, Rabbat C, Fok M, McAlister F and Garg AX: Chronic kidney disease and mortality risk: A systematic review. J Am Soc Nephrol 17: 2034-2047, 2006.

3. Meguid El Nahas A and Bello AK: Chronic kidney disease: The global challenge. Lancet 365: 331-340, 2005.

4. Reiser J and Sever S: Podocyte biology and pathogenesis of kidney disease. Ann Rev Med 64: 357-366, 2013.

5. Floege $\mathrm{J}$ and Amann K: Primary glomerulonephritides. Lancet 387: 2036-2048, 2016.

6. Dalla Vestra M, Masiero A, Roiter AM, Saller A, Crepaldi G and Fioretto P: Is podocyte injury relevant in diabetic nephropathy? Studies in patients with type 2 diabetes. Diabetes 52: 1031-1035, 2003.

7. Trivedi S, Zeier M and Reiser J: Role of podocytes in lupus nephritis. Nephrol Dial Transplant 24: 3607-3612, 2009.

8. Chronic Kidney Disease Prognosis Consortium, Matsushita K, van der Velde M, Astor BC, Woodward M, Levey AS, de Jong PE, Coresh J and Gansevoort RT: Association of estimated glomerular filtration rate and albuminuria with all-cause and cardiovascular mortality in general population cohorts: A collaborative meta-analysis. Lancet 375: 2073-2081, 2010.

9. Lv J, Xu D, Perkovic V, Ma X, Johnson DW, Woodward M, Levin A, Zhang and Wang H: Corticosteroid therapy in IgA nephropathy. J Am Soc Nephrol 23: 1108-1116, 2012.

10. Swamynathan SK: Krüppel-like factors: Three fingers in control. Hum Genomics 4: 263-270, 2010.

11. Mallipattu SK, Liu R, Zheng F, Narla G, Ma'ayan A, Dikman S, Jain MK, Saleem M, D'Agati V, Klotman P, et al: Kruppel-like factor $15(\mathrm{KLF} 15)$ is a key regulator of podocyte differentiation. J Biol Chem 287: 19122-19135, 2012.
12. Gray S, Feinberg MW, Hull S, Kuo CT, Watanabe M, Sen-Banerjee S, DePina A, Haspel R and Jain MK: The Krüppel-like factor KLF15 regulates the insulin-sensitive glucose transporter GLUT4. J Biol Chem 277: 34322-34328, 2002.

13. Fisch S, Gray S, Heymans S, Haldar SM, Wang B, Pfister O, Cui L, Kumar A, Lin Z, Sen-Banerjee S, et al: Kruppel-like factor 15 is a regulator of cardiomyocyte hypertrophy. Proc Natl Acad Sci USA 104: 7074-7079, 2007.

14. Mallipattu SK, Guo Y, Revelo MP, Roa-Peña L, Miller T, Ling J, Shankland SJ, Bialkowska AB, Ly V, Estrada C, et al: Krüppel-like factor 15 mediates glucocorticoid-induced restoration of podocyte differentiation markers. J Am Soc Nephrol 28: 166-184, 2017.

15. Li N, Zhang J, Yan X, Zhang C, Liu H, Shan X, Li J, Yang Y, Huang C, Zhang P, et al: SIRT3-KLF15 signaling ameliorates kidney injury induced by hypertension. Oncotarget 8: 39592-39604, 2017.

16. Faul C, Donnelly M, Merscher-Gomez S, Chang YH, Franz S, Delfgaauw J, Chang JM, Choi HY, Campbell KN, Kim K, et al: The actin cytoskeleton of kidney podocytes is a direct target of the antiproteinuric effect of cyclosporine A. Nat Med 14: 931-938, 2008.

17. Mundel P, Reiser J and Kriz W: Induction of differentiation in cultured rat and human podocytes. J Am Soc Nephrol 8: 697-705, 1997.

18. Yang SH, Choi JW, Huh D, Jo HA, Kim S, Lim CS, Lee JC, Kim HC, Kwon HM, Jeong CW, et al: Roles of fluid shear stress and retinoic acid in the differentiation of primary cultured human podocytes. Exp Cell Res 354: 48-56, 2017.

19. Livak KJ and Schmittgen TD: Analysis of relative gene expression data using real-time quantitative PCR and the 2(-Delta Delta C(T)) Method. Methods 25: 402-408, 2001.

20. Turner JE, Paust HJ, Bennstein SB, Bramke P, Krebs C, Steinmetz OM, Velden J, Haag F, Stahl RA and Panzer U: Protective role for CCR5 in murine lupus nephritis. Am J Physiol Renal Physiol 302: F1503-F1515, 2012.

21. Meng XM, Nikolic-Paterson DJ and Lan HY: TGF-beta: The master regulator of fibrosis. Nat Rev Nephrol 12: 325-338, 2016.

22. Cattran DC, Feehally J, Cook HT, Liu ZH, Fervenza FC, Mezzano SA, Floege J, Nachman PH, Gipson DS, Praga M, et al: Kidney disease: Improving global outcomes (KDIGO) glomerulonephritis work group. KDIGO clinical practice guideline for glomerulonephritis. Kidney Int Suppl 2: 139-274, 2012.

23. Coresh J, Selvin E, Stevens LA, Manzi J, Kusek JW, Eggers P, Van Lente F and Levey AS: Prevalence of chronic kidney disease in the United States. JAMA 298: 2038-2047, 2007.

24. Bommer J: Prevalence and socio-economic aspects of chronic kidney disease. Nephrol Dial Transplant 17 (Suppl 11): S8-S12, 2002.

25. Turner J and Crossley M: Mammalian Kruppel-like transcription factors: More than just a pretty finger. Trends Biochem Sci 24: 236-240, 1999.

26. Cohen CD, Klingenhoff A, Boucherot A, Nitsche A, Henger A, Brunner B, Schmid H, Merkle M, Saleem MA, Koller KP, et al: Comparative promoter analysis allows de novo identification of specialized cell junction-associated proteins. Proc Natl Acad Sci USA 103: 5682-5687, 2006.

27. Patrakka J, Kestilä M, Wartiovaara J, Ruotsalainen V, Tissari P Lenkkeri U, Männikkö M, Visapää I, Holmberg C, Rapola J, et al: Congenital nephrotic syndrome (NPHS1): Features resulting from different mutations in Finnish patients. Kidney Int 58: 972-980, 2000.

28. Mollet G, Ratelade J, Boyer O, Muda AO, Morisset L, Lavin TA, Kitzis D, Dallman MJ, Bugeon L, Hubner N, et al: Podocin inactivation in mature kidneys causes focal segmental glomerulosclerosis and nephrotic syndrome. J Am Soc Nephrol 20: 2181-2189, 2009.

29. Barisoni L and Mundel P: Podocyte biology and the emerging understanding of podocyte diseases. Am J Nephrol 23: 353-360, 2003.

30. Rogers RG and Otis JS: Resveratrol-mediated expression of KLF15 in the ischemic myocardium is associated with an improved cardiac phenotype. Cardiovasc Drugs Ther 31: 29-38, 2017. 